“ (C) 2017 IEEE. Personal use of this material is permitted. Permission from IEEE must be obtained for all other uses, in any current or future media, including

reprinting/republishing this material for advertising or promotional purposes, creating new collective works, for resale or redistribution to servers or lists, or reuse of any copyrighted component of this work in other works." 


\title{
Nonconvex Spectral Algorithm for Solving BMI on the Reduced Order $H_{\infty}$ Control
}

\author{
Y. Shi*, H. D. Tuan* and S. Su* \\ ${ }^{*}$ Faculty of Engineering and Information Technology, University of Technology, Sydney, Australia \\ Email: ye.shi@student.uts.edu.au, tuan.hoang@uts.edu.au,steven.su@uts.edu.au
}

\begin{abstract}
The design of reduced-order $H_{\infty}$ control can be transformed into an optimization problem with bilinear matrix inequality (BMI) constraints, which is an NP-hard problem. We propose a method to equivalently transfer the BMI constraint into a convex LMI constraint plus a matrix-rank constraint. The optimization with matrix-rank constraint is iteratively solved by a sequence of semidefinite programming (SDP) problems. Simulations on several benchmark systems show that our algorithm is practical and efficient.
\end{abstract}

Index terms - reduced-order $H_{\infty}$ control, bilinear matrix inequality (BMI), matrix-rank constraint, nonconvex spectral optimization

\section{INTRODUCTION}

A wide variety of system and control problems, such as optimal controller design, robust stability, pole placement in linear system theory can be reformulated as an optimization problem with linear matrix inequality (LMI) constraints [1], [2]. The LMI constraints are convex, which can be efficiently solved by interior-point methods in SDP [3], [4]. However, the optimization problems with nonlinear matrix inequality constraints are not easy to handle due to their noncovexity and potential nonsmoothness.

In control system, the design of reduced-order output feedback controller is a significant application, which in general is a nonconvex control problem and can be transformed into an optimization problem with bilinear matrix inequality (BMI) constraints [2]. In [5], [6], it has been shown that optimization problems with BMI constraints are NP-hard. By now, there exist several different approaches to solve the BMI problem. [7] proposed the Branch and Cut algorithms improved from the Branch and Bound Algorithm in some randomly generated problems. [8] proposed coordinate descent methods with some defined search directions, however the convergence was not guaranteed. By making use of the first-order perturbation approximation, a Path-Following method for solving BMI was proposed in [9]. [10] developed a local optimization with convex-concave matrix inequality constraints to deal with BMI optimization. Ordinarily, finding a global optimum in BMI optimization is impractical. Most of the global algorithms for BMI are limited to problems with small dimension [7].

In this paper, the BMI constraint is equivalently transferred to a LMI constraint plus a matrix-rank constraint, which is then expressed by spectral nonlinear function. Then our method is to solve a sequence of SDP problems to obtain the upper bound of the original BMI problem. The algorithm proposed in this paper is simple to implement by using SDP software tools, such as Sedumi [11] and SDPT3 [12].
The remainder of the paper is organized as follows. Section II is devoted to the Bilinear Matrix Inequality(BMI) formulation on structured $H_{\infty}$ control. Section III presents the nonconvex spectral optimization algorithms on $H_{\infty}$ control. Its computational simulation is provided in Section IV. The conclusions are drawn in Section V.

Notation. Notation used in this paper is standard. Particularly, $X \succeq 0, X \succ 0, X \preceq 0$ and $X \prec 0$ mean that a symmetric matrix $X$ is positive semi-definite, positive definite, negative semi-definite and negative definite, respectively. Trace $(X)$ represents the trace of $X$. In symmetric block matrices or long matrix expressions, we use $*$ as an ellipsis for terms that are induced by symmetry, e.g.,

$$
K\left[\begin{array}{cc}
S+(*) & * \\
M & Q
\end{array}\right] * \equiv K\left[\begin{array}{cc}
S+S^{T} & M^{T} \\
M & Q
\end{array}\right] K^{T}
$$

\section{BMI FORMULATION ON REDUCED ORDER $H_{\infty}$ CONTROL}

Consider a continuous time system

$$
\begin{aligned}
\dot{x} & =A x+B_{\infty} w+B u \\
z & =C_{\infty} x+D_{\infty} w+D_{\infty u} u \\
y & =C x+D_{y \infty} w
\end{aligned}
$$

where $x \in R_{x}^{n}$ is the system state, $u \in R^{n_{u}}$ is the system control, $y \in R^{n_{y}}$ is the measured output, $w \rightarrow z$ is the $H_{\infty}$ performance channel of the same dimension $n_{\infty}$.

We seek a structured output feedback control

$$
\left(\begin{array}{c}
\dot{x}_{K} \\
u
\end{array}\right)=\mathbf{K}\left(\begin{array}{c}
x_{K} \\
y
\end{array}\right), \mathbf{K} \subset R^{\left(n_{K}+n_{u}\right) \times\left(n_{K}+n_{y}\right)}
$$

where $n_{K}$ is the controller order, $\mathbf{K}$ is a convex structure such that

- The closed-loop system is internally stable

- $\quad$ The $H_{\infty}$-performance $T_{w \rightarrow z}$ is minimized

$$
T_{w \rightarrow z} \rightarrow \min
$$


Introduce the shorthands

$$
\begin{aligned}
& A_{0}=\left(\begin{array}{cc}
A & 0 \\
0 & 0_{k}
\end{array}\right) \in R^{\left(n_{x}+n_{K}\right) \times\left(n_{x}+n_{K}\right)}, \\
& B_{0, \infty}=\left(\begin{array}{c}
B_{\infty} \\
0
\end{array}\right) \in R^{\left(n_{x}+n_{K}\right) \times n_{\infty}}, \\
& C_{0, \infty}=\left(\begin{array}{ll}
C_{\infty} & 0
\end{array}\right) \in R^{n_{\infty} \times\left(n_{x}+n_{K}\right)}, \\
& \mathcal{B}=\left(\begin{array}{cc}
0 & B \\
I_{n_{K}} & 0
\end{array}\right) \in R^{\left(n_{x}+n_{K}\right) \times\left(n_{K}+n_{u}\right)}, \\
& \mathcal{C}=\left(\begin{array}{cc}
0 & I_{n_{K}} \\
C & 0
\end{array}\right) \in R^{\left(n_{K}+n_{y}\right) \times\left(n_{x}+n_{K}\right)} \text {, } \\
& \mathcal{D}_{12}=\left(\begin{array}{ll}
0 & D_{\infty u}
\end{array}\right) \in R^{n_{\infty} \times\left(n_{K}+n_{u}\right)}, \\
& \mathcal{D}_{21}=\left(\begin{array}{c}
0 \\
D_{y \infty}
\end{array}\right) \in R^{\left(n_{K}+n_{y}\right) \times n_{\infty}}, x_{c l}=\left(\begin{array}{c}
x \\
x_{K}
\end{array}\right) \text {. }
\end{aligned}
$$

The closed-loop system is

$$
\left(\begin{array}{c}
\dot{x}_{c l} \\
z_{\infty}
\end{array}\right)=\left(\begin{array}{cc}
A_{0}+\mathcal{B} \mathbf{K C} & B_{0, \infty}+\mathcal{B K}_{\mathbf{K}} \mathcal{D}_{2, \infty} \\
C_{0, \infty}+\mathcal{D}_{12} \mathbf{K C} & D_{\infty}+\mathcal{D}_{12} \mathbf{K} \mathcal{D}_{2, \infty}
\end{array}\right)\left(\begin{array}{c}
x_{c l} \\
w_{\infty}
\end{array}\right)
$$

The BMI formulation is following [2]

$$
\begin{aligned}
& \min _{\gamma, \mathbf{X}, \mathbf{K}} \gamma \quad \text { s.t. } \\
& \left(\begin{array}{ccc}
\left(A_{0}+\mathcal{B} \mathbf{K C}\right) \mathbf{X}+* & * & * \\
\left(B_{0, \infty}+\mathcal{B} \mathbf{K} \mathcal{D}_{2, \infty}\right)^{T} & -\gamma I & * \\
\left(C_{0, \infty}+\mathcal{D}_{12} \mathbf{K} \mathcal{C}\right) \mathbf{X} & D_{\infty}+\mathcal{D}_{12} \mathbf{K} \mathcal{D}_{2, \infty} & -\gamma I
\end{array}\right) \prec 0 \\
& \mathbf{X} \succ 0, \quad \gamma>0 .
\end{aligned}
$$

It is obvious that (5) is the minimization of linear objective function with BMI constraints (5a) and convex constraints (5b). It should be mentioned that, when the controller $\mathbf{K}$ is unstructured and full rank, optimization (5) is convex [13]. Introducing the new variable

$$
\mathbf{W}=\mathbf{K C} \mathbf{X}
$$

i.e.

$$
\mathbf{W} \in R^{\left(n_{K}+n_{u}\right) \times\left(n_{x}+n_{K}\right)},
$$

we can rewrite (5) by

$$
\begin{aligned}
& \min _{\gamma, \mathbf{X}, \mathbf{W}, \mathbf{K}} \gamma \quad \text { s.t., } \\
& \left(\begin{array}{ccc}
\left(A_{0} \mathbf{X}+\mathcal{B} \mathbf{W}\right)+* & * & * \\
\left(B_{0, \infty}+\mathcal{B} \mathbf{K} \mathcal{D}_{2, \infty}\right)^{T} & -\gamma I & * \\
C_{0, \infty} \mathbf{X}+\mathcal{D}_{12} \mathbf{W} & D_{\infty}+\mathcal{D}_{12} \mathbf{K} \mathcal{D}_{2, \infty} & -\gamma I
\end{array}\right) \\
& \mathbf{X} \succ 0, \quad \gamma>0, \\
& \mathbf{W}=\mathbf{K C X} .
\end{aligned}
$$

It can be seen that in optimization (7), (7a) and (7b) are convex constraints, however constraint $(7 \mathrm{c})$ is nonconvex. Now all the difficulty of BMI optimization (7) turns to the nonconvex constraint (7c), in the next section we will propose a nonconvex spectral algorithm to deal with the constraint (7c).

\section{Nonconvex Spectral Algorithm on $H_{\infty}$} CONTROL

To handle the nonconvex constraint $(7 \mathrm{c})$, let's begin with an auxiliary result.

Lemma 1: [14] For given matrix $W_{12}, W_{22}$ of sizes $n \times m$ and $m \times m$ with $W_{22}$ positive definite, one has

$$
\left(\begin{array}{ll}
0 & W_{12} \\
W_{12}^{T} & W_{22}
\end{array}\right) \geq 0
$$

if and only if $W_{12}=0$.

Using the above Lemma, we are now in a position to state the following result, which is a cornerstone in handling bilinear constraints like (6).

Theorem 1: The constraint (6) is equivalent to

$$
\begin{aligned}
\left(\begin{array}{lll}
\mathbf{W}_{11} & \mathbf{W} & \mathbf{K} \\
\mathbf{W}^{T} & \mathbf{W}_{22} & \mathbf{X}^{T} \mathcal{C}^{T} \\
\mathbf{K}^{T} & \mathcal{C X} & I
\end{array}\right) \geq 0 \\
\quad \operatorname{Trace}\left(\mathbf{W}_{11}-\mathbf{K K}^{T}\right) \leq 0 .
\end{aligned}
$$

Proof By Shur's complement, (9) is equivalent to

$$
\begin{aligned}
& \left(\begin{array}{cc}
\mathbf{W}_{11} & \mathbf{W} \\
\mathbf{W}^{T} & \mathbf{W}_{22}
\end{array}\right)-\left(\begin{array}{c}
\mathbf{K} \\
\mathbf{X}^{T} \mathcal{C}^{T}
\end{array}\right)\left(\begin{array}{ll}
\mathbf{K}^{T} & \mathcal{C X}
\end{array}\right)= \\
& \left(\begin{array}{cc}
\mathbf{W}_{11}-\mathbf{K K}^{T} & \mathbf{W}-\mathbf{K} \mathcal{C} \mathbf{X} \\
\mathbf{W}^{T}-\mathbf{X}^{T} \mathcal{C}^{T} \mathbf{K}^{T} & \mathbf{W}_{22}-\mathbf{X}^{T} \mathcal{C}^{T} \mathcal{C} \mathbf{X}
\end{array}\right) \geq 0,
\end{aligned}
$$

which implies $\mathbf{W}_{11}-\mathbf{K K}^{T} \geq 0$. This together with (10) yields $\mathbf{W}_{11}=\mathbf{K} \mathbf{K}^{T}$. Then applying Lemma 1 to (11) gives $\mathbf{W}=\mathbf{K C} \mathbf{X}$ as desired.

Similarly, by Shur's complement, and $\mathbf{W}_{11}-\mathbf{K K}^{T} \geq 0$, inequality constraint (10) can be equivalently replaced by

$$
\operatorname{rank}\left(\mathcal{W}_{K}\right)=n_{y}+n_{K}
$$

where

$$
\mathcal{W}_{K}:=\left(\begin{array}{ll}
\mathbf{W}_{11} & \mathbf{K} \\
\mathbf{K}^{T} & I_{n_{y}+n_{K}}
\end{array}\right)
$$

which is equivalent to

$$
\operatorname{Trace}\left(\mathcal{W}_{K}\right)-\lambda_{\left[n_{y}+n_{K}\right]}\left(\mathcal{W}_{K}\right)=0
$$

where $\operatorname{Trace}\left(\mathcal{W}_{K}\right)$ is the trace of $\mathcal{W}_{K}, \lambda_{\left[n_{y}+n_{K}\right]}\left(\mathcal{W}_{K}\right)$ is the summation of $\left(n_{y}+n_{K}\right)$ largest eigenvalues of $\mathcal{W}_{K}$.

Thus, the optimization problem (7) is equivalent to

$$
\min _{\gamma, \mathbf{X}, \mathbf{W}, \mathbf{K}} \gamma \quad \text { s.t. } \quad(7 b),(7 c),(9),(13)
$$

Instead of dealing with the nonconvex constraint (13) we incorporate it into the objective, leading to the following formulation (15),

$$
\begin{array}{ccc}
F_{\mu}:=\min _{\gamma, \mathbf{X}, \mathbf{W}, \mathbf{K}} & \gamma+\mu\left[\operatorname{Trace}\left(\mathcal{W}_{K}\right)-\right. \\
\left.\lambda_{\left[n_{y}+n_{K}\right]}\left(\mathcal{W}_{K}\right)\right] & \text { s.t. } & (7 b),(7 c),(9) .
\end{array}
$$

Where $\mu>0$ is a penalty parameter. Without squaring on the factor of $\mu$, the above penalization is exact, meaning that the constraint (13) can be satisfied by a minimizer of (14) with 
a finite value of $\mu$ (see e.g. [15, Chapter 16]). On the other hand, any feasible $(\gamma, \mathbf{X}, \mathbf{W}, \mathbf{K})$ to (14) is also feasible to (15), implying that the optimal value of (15) for any $\mu>0$ is upper bounded by the optimal value of (14).

Function $\lambda_{\left[n_{y}+n_{K}\right]}\left(\mathcal{W}_{K}\right)$ is nonsmooth but is lower bounded by

$$
\begin{aligned}
\lambda_{\left[n_{y}+n_{K}\right]}\left(\mathcal{W}_{K}\right) & =\sum_{i=1}^{n_{y}+n_{K}}\left(w_{i}\right)^{T} \mathcal{W}_{K} w_{i} \\
& \geq \sum_{i=1}^{n_{y}+n_{K}}\left(w_{i}^{(\kappa)}\right)^{T} \mathcal{W}_{K} w_{i}^{(\kappa)},
\end{aligned}
$$

where $w_{1}^{(\kappa)}, \ldots, w_{n_{y}+n_{K}}^{(\kappa)}$ are the normalized eigenvectors corresponding to $n_{y}+n_{K}$ largest eigenvalues of $\mathcal{W}_{K}^{(\kappa)}$.

Thus, any $(\gamma, \mathbf{X}, \mathbf{W}, \mathbf{K})$ feasible to the convex constraints (7b), (7c), the following convex optimization provides the upper bound for the nonconvex optimization (15),

$$
\begin{array}{r}
\min _{\gamma, \mathbf{X}, \mathbf{W}, \mathbf{K}} F_{\mu}^{(\kappa)}:=\gamma+\mu\left[\operatorname{Trace}\left(\mathcal{W}_{K}\right)-\right. \\
\left.\sum_{i=1}^{n_{y}+n_{K}}\left(w_{i}^{(\kappa)}\right)^{T} \mathcal{W}_{K} w_{i}^{(\kappa)}\right] \quad \text { s.t. } \quad(7 b),(7 c),(9) .
\end{array}
$$

because $F_{\mu}^{(\kappa)} \geq F_{\mu}$ according to (16). Therefore, for the optimal solution $\gamma^{(\kappa+1)}, \mathbf{X}^{(\kappa+1)}, \mathbf{W}^{(\kappa+1)}, \mathbf{K}^{(\kappa+1)}$ of the convex program (17), it is true that

$$
\begin{aligned}
& F_{\mu}\left(\gamma^{(\kappa+1)}, \mathbf{X}^{(\kappa+1)}, \mathbf{W}^{(\kappa+1)}, \mathbf{K}^{(\kappa+1)}\right) \leq \\
& F_{\mu}^{(\kappa)}\left(\gamma^{(\kappa+1)}, \mathbf{X}^{(\kappa+1)}, \mathbf{W}^{(\kappa+1)}, \mathbf{K}^{(\kappa+1)}\right) \leq \\
& F_{\mu}^{(\kappa)}\left(\gamma^{(\kappa)}, \mathbf{X}^{(\kappa)}, \mathbf{W}^{(\kappa)}, \mathbf{K}^{(\kappa)}\right) \\
& F_{\mu}\left(\gamma^{(\kappa)}, \mathbf{X}^{(\kappa)}, \mathbf{W}^{(\kappa)}, \mathbf{K}^{(\kappa)}\right),
\end{aligned}
$$

implying that $\left(\gamma^{(\kappa+1)}, \mathbf{X}^{(\kappa+1)}, \mathbf{W}^{(\kappa+1)}, \mathbf{K}^{(\kappa+1)}\right)$ is better than $\left(\gamma^{(\kappa)}, \mathbf{X}^{(\kappa)}, \mathbf{W}^{(\kappa)}, \mathbf{K}^{(\kappa)}\right)$ towards optimization (17).

The algorithm 1 is pseudo-code for the above procedure.

\section{Simulation RESUlTS}

The hardware and software facilities for our computational implementation are: (i) Processor: Intel(R) Core(TM) i5-3470 CPU @3.20GHz; (ii) Software: Matlab version R2015b; (iii) CVX with SDPT3 solver for SDP (17). The convergence stop criterion is set as $\epsilon=10^{-5}$.

To show the efficiency of our algorithm, helicopter system, chemical reactor system and transport airplane system are tested. All state space data of these systems are from [2].

In helicopter system, the sate dimension $n_{x}=4$, we seek to find a dynamic controller of order $n_{K}=2$, the algorithm successfully solve the problem, obtaining $\gamma=0.104$, compared with $\gamma=0.133$ in [13]. The controller

In chemical reactor system, the sate dimension $n_{x}=4$, we seek to find a dynamic controller of order $n_{K}=2$, the algorithm successfully solve the problem, obtaining $\gamma=1.037$, compared with $\gamma=1.142$ in [13].

In transport airplane system, the sate dimension $n_{x}=10$, we seek to find a dynamic controller of order $n_{K}=1$, the algorithm successfully solve the problem, obtaining $\gamma=1.237$, compared with $\gamma=2.860$ in [13].
Algorithm 1 Nonconvex Spectral Optimization Algorithm for Solving BMI on $\mathcal{H}_{\infty}$ control

1: Initialize $\kappa:=0$ and solve

$$
\min _{\gamma, \mathbf{X}, \mathbf{W}, \mathbf{K}} \gamma \quad \text { s.t. } \quad(7 b),(7 c),(9) \text {. }
$$

to find its optimal solution $\left(\gamma^{(\kappa)}, \mathbf{X}^{(\kappa)}, \mathbf{W}^{(\kappa)}, \mathbf{K}^{(\kappa)}\right)$, stop the algorithm if

$$
\operatorname{Trace}\left(\mathcal{W}_{K}^{(\kappa)}\right)-\sum_{i=1}^{n_{y}+n_{K}}\left(w_{i}^{(\kappa)}\right)^{T} \mathcal{W}_{K}^{(\kappa)} w_{i}^{(\kappa)} \leq \epsilon
$$

and accept $\left(\gamma^{(0)}, \mathbf{X}^{(0)}, \mathbf{W}^{(0)}, \mathbf{K}^{(0)}\right)$ as the optimal solution of the nonconvex program (14).

2: repeat

3: Solve the convex program (17), to find the optimal solution $\left(\gamma^{(\kappa+1)}, \mathbf{X}^{(\kappa+1)}, \mathbf{W}^{(\kappa+1)}, \mathbf{K}^{(\kappa+1)}\right)$

4: $\quad$ Set $\kappa:=\kappa+1$.

5: until

$$
\operatorname{Trace}\left(\mathcal{W}_{K}^{(\kappa)}\right)-\sum_{i=1}^{n_{y}+n_{K}}\left(w_{i}^{(\kappa)}\right)^{T} \mathcal{W}_{K}^{(\kappa)} w_{i}^{(\kappa)} \leq \epsilon
$$

6: Accept $\left(\gamma^{(\kappa)}, \mathbf{X}^{(\kappa)}, \mathbf{W}^{(\kappa)}, \mathbf{K}^{(\kappa)}\right)$ as a found solution of (14).

The detail computational results are summarized in Table I, where the first column is the cases name, the second column is the state space dimension, the third column is the value of $\mu$. The forth column is the found value of $\gamma$. The fifth column is the iterations by our method; the $\gamma$ found in [13] is provided in the sixth column.

TABLE I: Numerical results compared with [13]

\begin{tabular}{|c|c|c|c|c|c|}
\hline Cases & {$\left[n_{x}, n_{y}, n_{u}, n_{K}\right]$} & $\mu$ & $\gamma$ & $\#$ iter & $\gamma$ in [13] \\
\hline Helicopter & {$[4,1,2,2]$} & 1 & 0.104 & 2 & 0.133 \\
\hline Chemical reactor & {$[4,3,2,2]$} & 2 & 1.037 & 4 & 1.142 \\
\hline Transport aircraft & {$[10,3,2,1]$} & 2 & 1.237 & 5 & 2.860 \\
\hline
\end{tabular}

Above all the cases tested, we can see that the optimal values reported by our algorithm are better than [13]. And it takes only a few iterations to converge.

\section{CONCLUSION}

We have proposed a new algorithm for solving BMI optimization arising in $H_{\infty}$ control. Unlike the previous developments, we equivalently transferred the BMI constraint into a convex LMI constraint plus a matrix-rank constraint. The optimization with matrix-rank constraint can be iteratively solved from a sequence of SDP problems. The numerical results on several benchmark systems have shown that our algorithm is practical and efficient. The extension to mix $H_{2} / H_{\infty}$ controller design is currently under development.

\section{REFERENCES}

[1] S. Boyd, L. El Ghaoui, E. Feron, and V. Balakrishnan, Linear matrix inequalities in system and control theory, vol. 15. SIAM, 1994. 
[2] F. Leibfritz, "Compleib: Constraint matrix optimization problem library a collection of test examples for nonlinear semidefinite programs, control system design and related problems," tech. rep., Univ. Trier, Dept. Math, Trier, Germany, 2004.

[3] Y. Nesterov and A. Nemirovskii, Interior-point polynomial algorithms in convex programming, vol. 13. SIAM, 1994.

[4] L. Vandenberghe and S. Boyd, "Semidefinite programming," Society for Industrial and Applied Mathematics, vol. 38, pp. 49-95, March 1996.

[5] O.Toker and H.Ozbay, "On the NP-hardness of solving bilinear matrix inequalities and simultaneous stabilization with static output feedback," in American Control Conference, pp. 2525-2526, 1995.

[6] J. G. VanAntwerp and R. D. Braatz, "A tutorial on linear and bilinear matrix inequalities," Journal of Process Control, vol. 10, pp. 363-385, 2000.

[7] M. Fukuda and M. Kojima., "Branch-and-cut algorithms for the bilinear matrix inequality eigenvalue problem," Computational Optimization and Applications, vol. 19, pp. 79-105, 2001.

[8] T. Iwasaki, "The dual iteration for fixed-order control," IEEE Transactions on Automatic Control, vol. 44, no. 4, pp. 783-788, 1999.

[9] A. Hassibi, J. How, and S. Boyd, "A path-following method for solving bmi problems in control," in American Control Conference, pp. 13851389, 1999.

[10] Q. T. Dinh, S. Gumussoy, W. Michiels, and M. Diehl, "Combining convex-concave decompositions and lienarization approaches for solving BMIs, with application to static outputfeedback," IEEE Trans. Automat. Control, vol. 57, pp. 1377-1390, 2012.

[11] J. F. Sturm, "Using SeDuMi 1.02: A matlab toolbox for optimization over symmetric cones," Optim. Methods Software, vol. 11-12, pp. 625653, 1999.

[12] R. H. Tütüncü, K. C. Toh, and M. J. Todd, "Solving semidefinite quadratic linear programs using SDPT3," Mathematical programming, vol. 95, pp. 189-217, 2003.

[13] P. Apkarian, D. Noll, and O. Prot, "A trust region spectral bundle method for nonconvex eigenvalue optimization," SIAM Journal on Optimization, vol. 19, no. 1, pp. 281-306, 2008.

[14] U. Rashid, H. D. Tuan, and H. H. Nguyen, "Joint optimization of source precoding and relay beamforming in wireless MIMO relay networks," IEEE Trans. on Commun., vol. 62, pp. 488-499, 2014.

[15] J. F. Bonnans, J. C. Gilbert, C. Lemarechal, and C. Sagastizabal, Numerical Optimization Theoretical and Practical Aspects (second edition). Springer, 2006. 